\section{Replicating The Unreplicatable}

Charles A. Garber, Ph.D., SPI Supplies

When doing replication, it is very important that all traces of the sample be removed from the replica. Adhering sample material introduces artifacts into the image and may, in extreme cases, make the replica so thick that the TEM beam cannot "see" through it. This may be a problem for both "ordinary" Pt/C replicas and freeze fracture situations. For organic samples, including both biological materials and polymers. For example, polymer latex particle fragments have a particular tendency to stick to the replica in freeze fracture preparations. Some freeze fracture samples will "wet" the replica surface if the replica is stripped by melting the substrate.

The typical protocol calls for removal of these residues by a strongly acidic chemical oxidizing process, for example one using a chromic/sulfuric acid mixture. Yes, these baths will remove organic materials, but they pose hazards to the people in the laboratory, they have disposal problems and the surface tension forces of the liquid have a tendency to disrupt the fine texture and pores which one is attempting to resolve. We have found that an alternate approach is easier and safer to use as well as being less damaging to the replica:

1) After replication with $\mathrm{Pt/C}$ (or in extreme cases, only Pt), the replica is "backed" with a layer of silicon oxide, deposited in a vacuum evaporator by the evaporation of silicon monoxide (SiO) from a tungsten basket. If the preparation is being done within a freeze fracture apparatus, then an additional set of "posts" would be required to follow the Pt/C with the SiO coating

2) Do your best to strip the inorganic replica from the organic sample; you have probably already learned how to do this for your particular samples prepared in the way you prepare them.

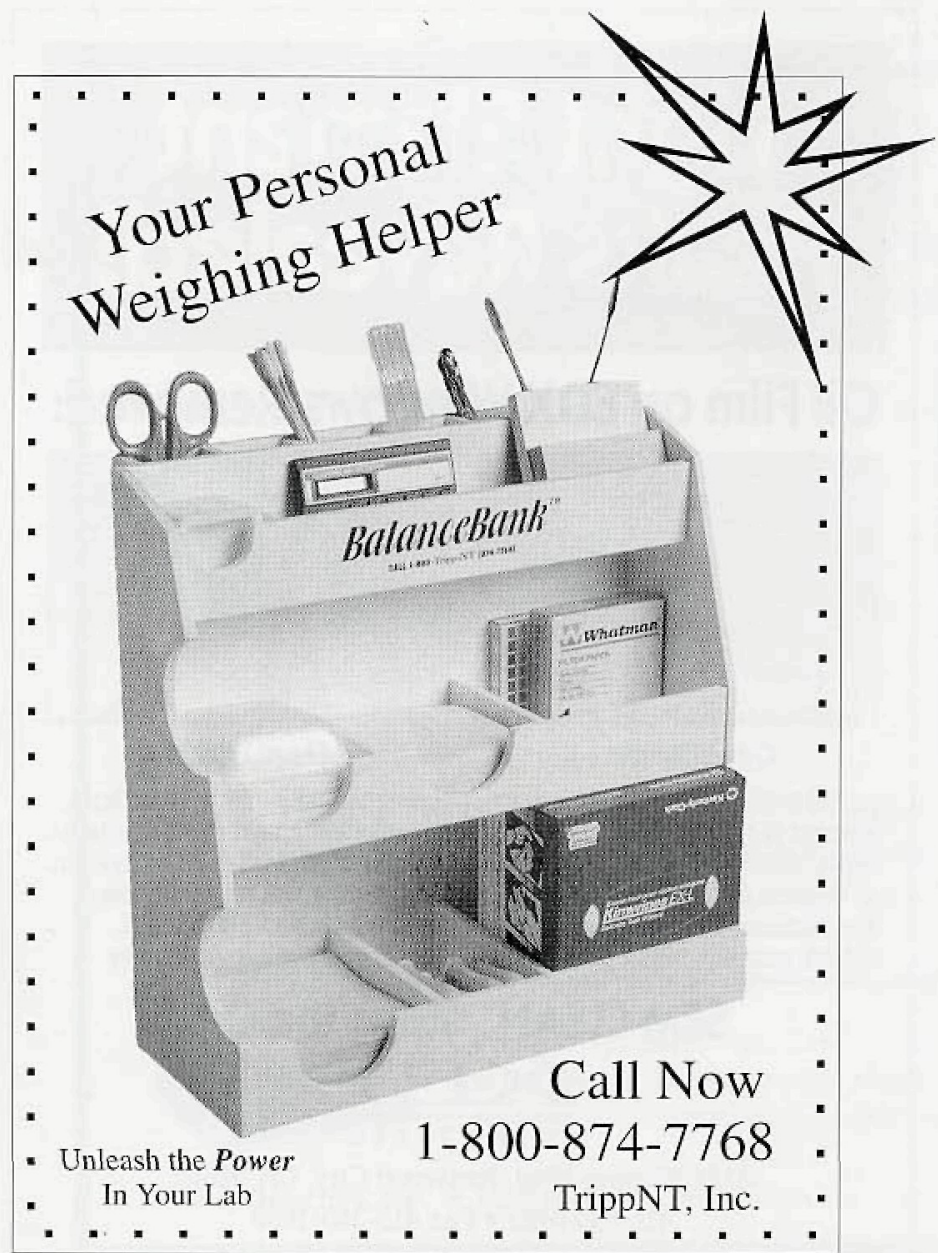

3) Put the remaining replica into a plasma etcher and etch using oxygen only to removed all remaining organic residues. Typical etching times are less than one minute and may be as little as 10 seconds. This procedure may attack the carbon replica film enough that it may affect the coherence of the replica, and this is the reason for the silicon oxide "backing" layer, which will not be affected by oxygen plasma

4) The replica is put onto a TEM grid, and one would hope to "see" the features of interest. The low atomic numbers of silicon and oxygen, like that of carbon, mean that there should not be any meaningful loss of contrast. Since the grain size of Pt in Pt/C replication is typically less than $1 \mathrm{~nm}$, pores as small as $10 \mathrm{~nm}$ should be resolved without artifacts.

If it does not work the first time, not to worry. Your first Pt/C replica didn't work either, did it? In fact, if it worked the first time, you probably did something wrong! This is a delicate procedure, and you will need to do appropriate validation for your particular kinds of samples.

If you are not experienced in the deposition of silicon oxide films, you may be concerned about artifacts. Yes, there are artifacts in silicon oxide films, just as there are artifacts in carbon films. They are controlled in the same way, by practice in making replicas and examining the replicas. In a platinum-shadowed image, however, the low atomic numbers of carbon, oxygen and silicon generally make the artifacts fade into the background of the platinum-shadowed image.

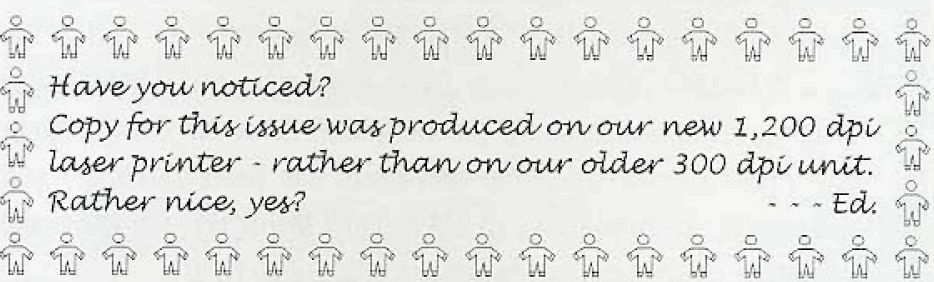

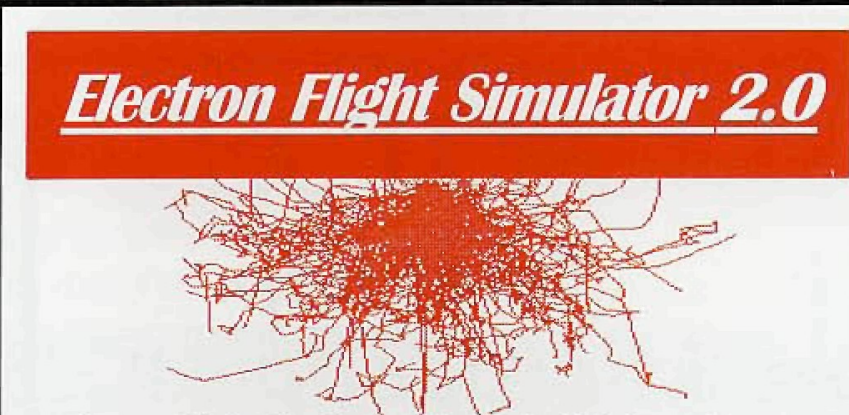

Electron Flight Simulator, software for Windows, lets you model your sample and electron microscope conditions so you can see where your $x$-ray signal is coming from. Have more confidence in your analysis with less guesswork. $\mathrm{X}$-ray analysis will never be the same.

- Model a Bulk, Thin Film, or Film on a Substrate

- Model up to Five Layers, Alone or on a Substrate

- Model any Sample Chemistry at any Accelerating Voltage

- Model Particles on a Sample or Inclusions in a Sample

- Model Vertical Interfaces Between Layers

- Model any Sample Tilt from 0 to 90 Degrees

- Display PhiRhoZ Depth of X-ray Generation Plots

- Display X-ray and Electron Interaction Volumes

- Display Electrons by Color Change at Each $10 \%$ Energy Loss

- Print Your Models or Save Them as Image Files

- On-Line Help Menus

- On-Line Periodic Table and Chemistry Calculator TM

- Unlimited Free Technical Support

- The Perfect Teaching and Training Tool

\section{Small World \\ P.O. Box 25284 San Mateo, CA 94402 \\ Phone/Fax (415) 345-8013 \\ e-mail: DCHERNOFF @ AOL.COM}


$\checkmark$ Oct 12/14 '95: Great Lakes Microscopy Conference '95. (GLeMA) Toledo, OH. Dr. Carol Heckman: (419)372-8218

$\checkmark$ Oct 16/20 '95: AVS Annual National Symposium. Minneapolis, MN. Tel: (212)248-0200, Fax: (212)248-0245.

$\checkmark$ Oct 17/20 95: Scanning Electron Microscopy and X-Ray Microanalysis for the Material Scientists. (SUNY) New Paltz, NY (914)255-0757, Fax: (914)255-0978

$\checkmark$ Oct 18 95: Short Course on Image Analysis (Umanalco). lowa City, IA. Tel./Fax:

(319) $337-2474$.

$\checkmark$ Oct 24/27 95: Ultramicrotomy in Materials Science (RMC and Univ. of Arizona). Tucson, AZ. Bob Chiovetti: (520)889-7900, Fax: (520)741-2200.

$\checkmark$ Oct 25/Nov 1 95: Optical Microscopy and Imaging in the Biomedical Sciences (Marine Biology Lab). Woods Hole, MA. (508)548-3705, eMail: admissions@mbl.edu

$\checkmark$ Nov 10/11 95: Tripod Polisher Workshop (South Bay Technology). San Clemente, CA. (800)SBT-2233, Fax: (714)492-1499.

$\checkmark$ Nov 15 95: 23rd Scottish Microscopy Symposium. Edinburgh, Scottland. Stephan Helfer: 0131 552-7171, Fax: 0131 552-0382, Stephan@rbge.org.uk

$\checkmark$ Nov 28/Dec 1 '95: 34th Annual Conference of the Electron Microscopy Society of Southern Africa (EMSSA '95). Prof. Mike Lee, EM Unit, Univ. of the North, Private Bag X1106, Sovenga 0727, South Africa. email: qemssa95@uninl.north.ac.za $\checkmark$ Jan. 25/27 '96: Microwave Workshop. (Ted Pella, Inc.) San Diego, CA. Rick Gilberson: (800)637-3526 (CA) or (800)237-3526 (outside

CA).

$\checkmark$ 5/9 Feb '96: 14th Australian Conference on Electron Microscopy (ACEM-14) \& 1st Meeting of the International Union of Microbream Analysis Societies (IUMAS). Sydney, Australia. Maret Vesk: 61-2-3512351, Fax: 61-2-552-1967, eMail: maret@emu.su.oz.au

$\checkmark$ 3/8 March '96: Pittcon '96. Chicago, IL (412)825-3220, Fax: (412)825-3224

$\checkmark$ March 18/22 \& March 25/29 "96: Practical Aspects of Scanning Electron Microscopy (PASEM 96). (Univ of MD). Tim Maugel: (301)405-6896, Fax: (301)314-9358

\section{$\checkmark$ April $9 / 12$ ' 96 : SCANNING ' 96 .}

(Foundation for Advances in Medicine and Science, Inc.) Monterey, CA. Mary K. Sullivan: ((201)818-1010, Fax: (201)818-0086, eMail: fams@holonet.net

$\checkmark$ April 21/25 '96: 18th International Conference on Cement Microscopy. (ICMA) Houston, TX. Louis A. Jany: (610)926-1024, Fax: (610)926-1906/

$\checkmark$ May 11/16 '96: Scanning Microscopy, Cells and Materials, and food Structure 1996 Meeting (Scanning Microscopy International). Bethesda, MD. (708)529-6677, Fax: (708) $980-6698$.

$\checkmark$ June 4/7 '96: Protocols in Microscopic Imaging, Immunocytochemistry and Image Analysis. (Geo. Washington Univ.) Washington, D.C. Fred G. Lightfoot: (202)994-2881, Fax: (202)994-8885. $\checkmark$ June 10/21 '96: Lehigh University Microscopy Short Courses. Bethlehem, PA. Prof. David Williams: (610)758-5133, Fax: (610)7584244, eMail: interSEM@lehigh.edu

$\checkmark$ June 24/28 '96: 12th Annual Short Course on Molecular Microspectroscopy. (Miami Universtiy). Miami University: (513)529-2874, Fax (513) $529-7284$

$\checkmark$ July 2/4 '96: MICRO '96 (RMS), London, U.K. 441865248768 , Fax: 441865791237

$\checkmark$ July 4/19 '96': 43rd International Field Emission Symposium. Moscow, Russia. Prof. Alesander L. Suvorov: (095)12596 91/(095)12534 39, Fax: (095)34 39, eMail: surorov@cl itep.ru

$\checkmark$ July 27/August 4 '96: 3D Microscopy of Living Cells (Univ. of British Columbia). Vancouver, BC, Canada. Dr. James Pawley: (608)2633147, Fax: (608)265-5315, eMail: JPAWLEY@macc.wisc.edu

$\checkmark$ Aug '96: 6th Asia-Pacific Conference on Electron Microscopy, APEM 6. Hong Kong. Dr. E.C. Chew: 852609 6845, Fax: 8526035031

$\checkmark$ 8/17 August '96: 17 th Congress and General Assembly of the International Union for Crystallography. Seattle, WA. Prof. R.F. Bryan, Univ of $V A$.

$\checkmark$ 11/15 August ' 96 : MSAMAS/MSC Joint Annual Meeting. Minneapolis, MN MSA Business Office: (508)540-5594/(800)538-3672, Fax: (508)548-9053.

$\checkmark$ 26/30 August '96: EUREM '96. University College, Dublin, Ireland. Prof. Martin Steer: 353-17062254

$\checkmark 26 \mathrm{Sept} / 2$ Oct. '98: 14th International EM Congress. Cancun, Mexico. Miguel Jose Yacaman: Tel./Fax: 525-570-85-03

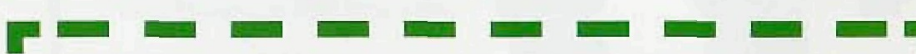 \\ NORAN/Iracor Northern EDS Repair}

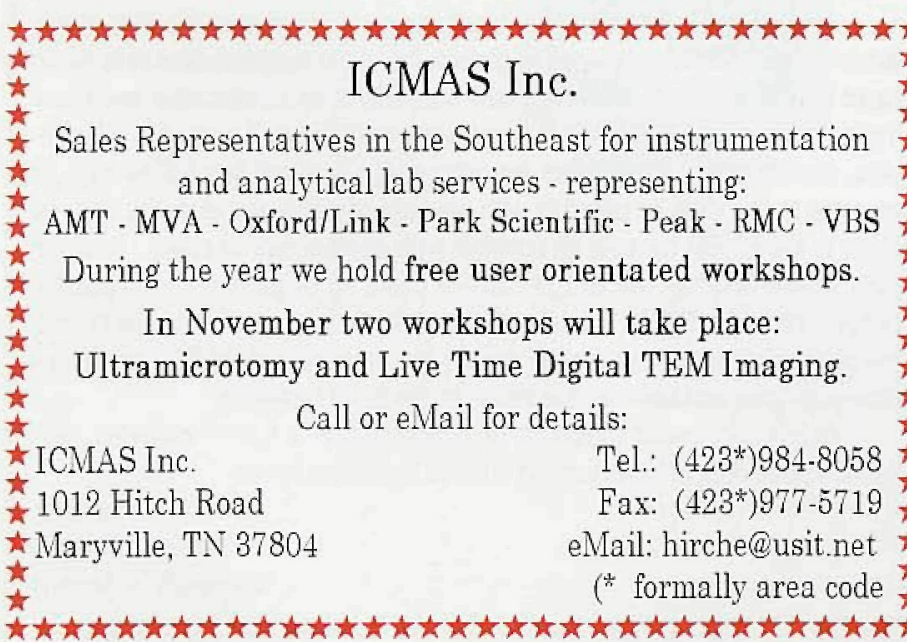

Factory and field trained personnel with over 35 years total experience, located in the the Midwest, Southeast and on the West Coast, specializing in TN2000 and TN5500 repair, detector upgrades, data storage, imaging hardware and peripheral output devices - and used equipment resale.

Choose from a full maintenance or parts only contract, or on-demand service - at rates normally a fraction of others

For further information, contact Doug Connors at:

\section{T Analyzer Service}

7897 Highway 19

Dane, WI 53529
Tel.: (608)798-2005

Fax: $(608) 798-1675$ 


\section{SERVICE IS OUR STrovg SuIT}
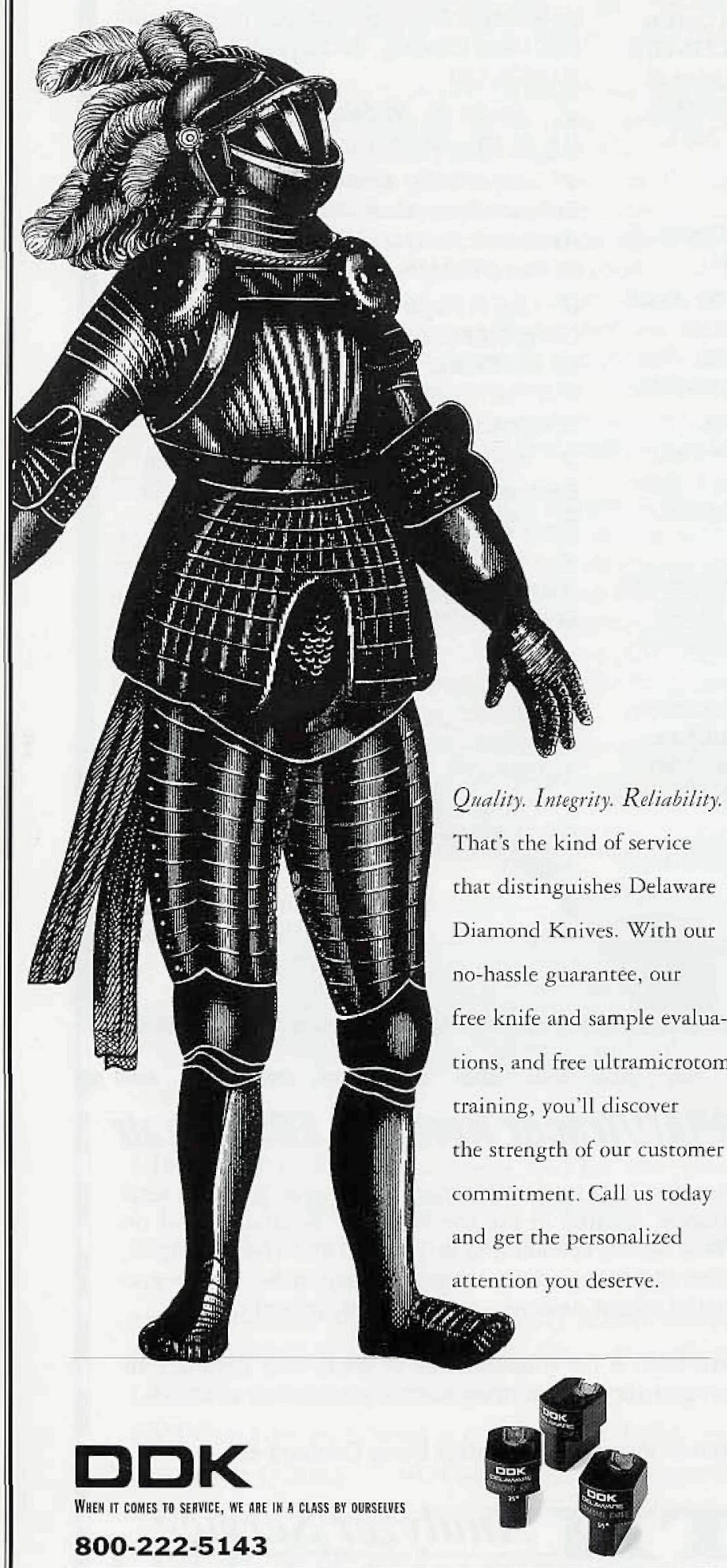

Delaware Diamond Knives, Inc.

3825 Lancaster Pike, Wilmington, DE 19805

Fax: 302-999-8320 E-Mail: DDKJOE@aol.com

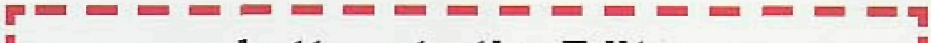

\section{Letters to the Editor}

Dear Don,

I have enjoyed reading the series of articles in your newsietter by Mox- I

tek relating to "More Than One Ever Wanted To Know About X-ray Detec- I tors" - the first six of the series by Dr. Mark Lund and the last by Dr. D. Clark Turner

IIn the last of the series, "If I Know It's There, Why Can't I see It?", Dr. I

Turner mentioned three alternatives to get around the problem of detecting trace amounts of copper in an aluminum film, but did not mention a fourth

I alternate: analysis by WDS. Resolution and sensitivity with WDS is approxi-

【 mately 10 times better than with EDS, therefore WDS could easily detect ॥ 0.5-1.0\% copper in aluminum. With WDS it is possible to analyze copper L $\alpha$ with excellent sensitivity, which means the accelerating voltage could be re-

duced to $10 \mathrm{keV}$ or less (the copper $\mathrm{L}$ absorption edge is $0.953 \mathrm{keV}$ ). This

I confines the beam to the aluminum film and prevents $X$-ray production from the silicon substrate.

From Dr. Clark's article, some of your readers might infer that $X$-ray

I microanalysis using a scanning electron microscope is not an appropriate technique for solving this problem. In fact, with the proper instrumentation this problem could be easily solved in the SEM lab.

I

Yours truly,

】

Is/ Joseph Carr

【

Microspec Corporation

Dear Don,

I would wanted to comment on the recent article in your publication "A Novel approach in Rembedding Thick Resin Sections for Ultramicrotomy". I

$\llbracket$ have several observations from my own experience.

This technique also works for embedding paraffin sections that have unique features that need to be studied by EM. Scribe on the back of the 1 slide (a diamond pen works well) the area to be re-embedded. Dissolve the I mounting media for the coverslip (depending on how fresh the mounting me- dia is, this may take several days of leaving the slide in Xylene). Glue the BEEM capsule over the area of interest (see comments below about other glue options). Re-hydrate the tissue from $100 \%$ ethanol to a buffered solution using the well created by the glued on BEEM capsule. Osmicate, dehydrate and embed (because of the thinness of the section, solution changes can be shortened)

I Another option for gluing the BEEM capsule down is "super glue". Put ] a small amount on some glassine weighing paper and using the end opposite from the tip (you want to use the smoothest surface, not the one you cut with

I the razor blade), drag it through the glue and set it in place on the slide. After I it dries it should be well sealed, although it may need a touch up if you were not generous with the glue. Fingernail polish works too (although I've never tried it with propylene oxide).

1 Our experience with the "pop off" technique led us to use harder plas- I tics, e.g., epons or their equivalent, rather than Spurrs. The medium hardness or slightly harder worked better for us.

I Another option for getting the block separated from the slide that avoids the annoying problem of pieces of glass adhering to the blockface is to avoid liquid $\mathrm{N}_{2}$ and use heat. We would take our slide/block combination and place

it slide down on an 80 degree $F$ (approximate) hot plate for roughly 3-5 min-

\utes. (Safety note: This makes everything HOT!). Grab the BEEM capsule 』 as close to the slide as possible with one pair of pliers and grab the slide as close to the BEEM capsule as possible with another pair of pliers. Hold the

I slide steady and turn the BEEM capsule pliers 90 degrees so as to pull the I tissue face of the BEEM capsule off the slide. We never had problems with the glass sticking and we almost always got the whole block face off except

where the glue had been on the inside of the BEEM capsule.

1 Appropriate credit should go to Charles Bjore, a former coworker, who I spent a good deal of time working out this technique for us.

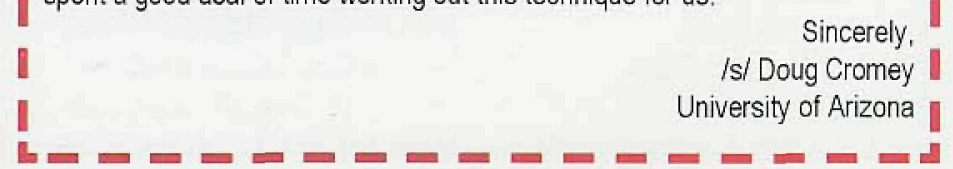

